\title{
10
}

\section{School Management Information System Authority in Israel}

\author{
Ilana Sagi, Yaffa Gev and Ahuva Feinmesser \\ Ministry of Education, Culture and Sports, Jerusalem, Israel
}

\begin{abstract}
The management of Israel's educational system entails the involvement of several entities - the Ministry of Education, local authorities, schools, training institutions, etc. - in terms of operation and responsibility. This is a system with a wide geographical distribution, which affects the country's entire population.

In order to overcome the complex and problematic nature of information in educational systems, it is necessary to enable integration and compatibility of the various systems, while at the same time leading to improvement of management and decision-making processes.

The end unit of this system is the SMIS - School Management Information System.

The objective of the SMIS is to improve administrative and pedagogic management and decision-making processes in schools by:

* Compiling and analyzing data

* Locating and displaying exceptional cases

* Increasing efficiency and reducing the administrative burden

* Providing tools to assist in the administrative and pedagogic decision-making process

Moreover, SMIS aims to link the school information system to the Ministry of Education and external information systems to constitute an infrastructure database enabling data flow in all school information systems.
\end{abstract}

Keyword Codes: K.3.1; K.4.3; K.6.1

Keywords: Computer uses in Education; Organizational impacts; Governmental issues; project and People Management.

\section{BACKGROUND}

The changes in information technology, i.e. miniaturization, reduced costs, simplification of use and maintenance, user friendliness and communicative effectiveness have led to a rapid development of school information systems. The importance of SMIS is greater in schools than in other organizations because of the uncertainty level in school management [1]. The more people recognize the benefits of information technology, the more aware they become of the need to adapt organizations and their administration to advanced technological methods. 


\section{SMIS OBJECTIVES}

The objective of SMIS is to improve administrative and pedagogic management and decision making processes in school. The school generates and uses data derived from such sources as parents, teachers, educational authorities and of course pupils. As the quantity of data is enormous, it is essential to have a tool to compile and to analyze data. When data is analyzed it is possible to locate and display exceptional cases. The IT provides tools to assist in the administrative and pedagogic decision making process. It increases efficiency and reduces the administrative burden. The build up of an infrastructural data base enables data to flow in all school information systems and enables linkage among school of Education, and other external information systems.

\section{SMIS AUTHORITY}

In 1989, the Ministry of Education initiated the SMIS project and the SMIS authority was established [2]. The SMIS authority is functioning as a "National Operations Center", to guide and monitor SMIS development and implementation. Its main functions and areas of responsibilities are: (a) development and maintenance of a standardization file, establishing a common data dictionary for all schools and software developers, supervising the software suppliers; (b) development and maintenance of a training course array; (c) development and maintenance of professional guidance array; (d) communication and contacts with other educational systems, local authorities and governmental authorities; (e) support and consultation; establishing an information center, assisting in implementation and integration of all factors in and out of schools.

\section{STANDARDIZATION FILE}

The standards for the software elements [3] - data, files, interface, etc.- are compiled into a dynamic file updated according to needs arising from procedures, feedback from schools and users, and requirements from the Ministry of Education. The file is updated twice a year followed by an implementation check-up.

The standardization file main parts are: (a) a basic system with basic modules for the school level system, and (b) guidelines for supplier services and maintenance.

\subsection{Basic Modules for SMIS Systems}

SMIS was developed as a platform software, i.e., a basic software kernel to which various modular components can be appended after acquiring the basic "MANBAS" (as SMIS is known in Israel) software. Each school can supplement it with add-on in a variety and formats suiting its needs and budget.

The basic software comprises the following modules:

Student data management

Teacher management

Subject management

Achievement recording and report

Pedagogic module - statistical analysis and reports

The guiding principle of standardization is that any software (besides the SMIS) to be added to the school in future will obtain student and teacher data from the basic system. 
Besides the above mentioned modules, there also add-on modules, i.e., self-evaluation system, curriculum and teaching aid management, bookkeeping, library-management, etc.

\subsection{Guidelines for Supplier Service and Maintenance}

A very important part of the standardization file is the module for guidelines and maintenance. It describes the methods for services and maintenance of procedures and software. It comprises the following subjects: (a) individual operational instruction in schools, provided in stages in accordance with the present stage of the school, (b)service and maintenance for hardware and software, and (c) software version updates.

To date, five software suppliers have received a franchise to operate the software in schools. The SMIS Authority maintains a control array on SMIS suppliers in all areas pertaining to application of standardization, compliance with standards and service levels, as well as handling incoming complaints from schools. If any SMIS supplier does not comply with the conditions stated above, the Ministry of Education can revoke its franchise for the sale of software.

\section{SMIS SEMINARS}

The SMIS authority performs a large training activity throughout the country. The training activity is divided into two major types: (a) seminars, (b) training and consultancy.

The seminars aim to cover SMIS introduction to the school management staff: principals and SMIS - coordinators on one hand and on the other hand to introduce and train SMIS school secretaries [4,5,6,7]. A 40-hours course was offered for the school management staff, and a 20 hours course for the secretarial staff.

The School's head - master attitude to SMIS implementation and its integration into school routine is extremely important, therefore the emphasis in the seminars is on these matters.

The objectives of the seminars are;

(1) Preparation of the background for receiving the SMIS

(2) Recognition of its necessity

(3) Training of functionaries

(4) Developing skills in the use of SMIS reports

(5) Increasing the efficiency of the decision-making process on the administrative and pedagogic level

The seminars' syllabus [8] includes: computerized information systems, introduction of modifications to the Protection of Privacy Law,, statistical concepts, introduction to SMIS products and their use, word processing, inculcation of additional skills - familiarization with the computer and experience with the software purchased by the relevant local authority. Seminars are administered within the region, on the basis of an agreement between the Ministry and the local authority. Each course consists of several sessions; each session includes lectures, workshops and practice.

SMIS courses and seminars are being given, initially to school principals and coordinators on one hand, and to school secretaries on the other, parallel to the introduction of the systems into schools. At a later stage, the objective is to instruct all teachers by means of institution-wide courses.

In view of the more widespread use of SMIS systems in schools within outlying settlements and the need for courses throughout Israel, it has been decided to expand the course frameworks to colleges and institutional entities. In order for those entities to benefit from the total experience accumulated by the SMIS authority, an Instruction Kit has been 
developed. The Instruction Kit includes a Lecturer's Workbook and a Student's Workbook (Manbas Authority 1992). The first contains a lesson plan, a detailed listing of lectures, and transparencies, while the second contains transparencies of the lectures with explanations and space for the student's comments.

\section{GUIDANCE AND IMPLEMENTATION}

The true test of the SMIS is its implementation or even assimilation- i.e., the use of its output in administrative and pedagogic decision-making, and its transformation into a vital and integral part of the school.

Introduction of SMIS systems into the schools is backed by close individual guidance. Internalization of the system into the school is affected to no small degree by the quality of guidance provided. For this reason, great resources and efforts are invested in guidance, and in the training and operation of a skilled team of consultants.

The role of the consultant in the implementation set-up is a crucial one. In some places, where guidance was not provided, the system stopped functioning after a while, or the tool was not used beyond producing students and teachers lists.

The consultant's role starts even before the installation of the computerized system in school. His task is to diagnose the stage of the absorption process of a school, in cooperation with the school principal. Together they build a plan of operation including written goals and timetable. The consultant supports the principal, helps-out the school SMIS coordinator in the initial stages, and teaches staff in subsequent stages by means of consultancy and advice in finding solutions. In many cases the consultant is liasing among the entities involved in operating SMIS, i.e., local department of education, suppliers, etc.

The consultant must provide encouragement and consultancy for the administrative/pedagogic staff, must acquaint them with the wide range of possibilities and options for use of the tool, and allow them to benefit from his experience. The consultant does not decide for the school; decisions are made by school functionaries.

The SMIS authority operates and supervises some 50 consultants. Some of these are employed by the local authorities, and others by the SMIS authority. All of the guidance activities, down to the most individual level, are reported to the SMIS authority through consultant's reports and input into the database for follow-up and control and problem solving. The data base comprises information of the degree of internalization. The SMIS authority, supports the local authorities. In order to do this the authority maintains a support center to which any question concerning the SMIS may be addressed - from requests for guidance in operating the software, through complaints against suppliers, and up to wishes for software development. The authority initiates activities to encourage the use of the SMIS, such as the publication of booklets and information sheets and organizing conferences. It moreover initiates study days on special subjects requiring intensive guidance and briefing, such as selfevaluation (computerized examinations), student roster, etc.

A procedure was established for the implementation of SMIS. This procedure contains mile-stones for monitoring the implementation process.

The first step in the implementation process is setting up a student file this is done either by keying-in the computer data from existing forms or by transferring a file from the local authority or from the ministry. In any event, data are checked and verified. A parallel step is setting up a teacher file which is done more or less in the 
same way as the student-file. Next step is the assignment to homeroom classes and study groups and setting up subject tables. The products of these stages are usually variou lists of students and teachers and other reports deriving from the student and teacher data-base, some schools implement the discipline and attendance module and sent reports to the parents.

The following step, which is regarded, as major step forward is the implementation of the pedagogic module. This step includes keying-in grades: term grades, test/term paper grades. Some schools enter this stage by starting to key-in grades of particular subjects throughout school, other gather grades of particular classes. Most schools begin by keying-in term grades in order to produce report cards. In the advance stages a school generates statistical reports which are used by the school principal, teachers, and pedagogic council.

System operation is implemented by the schools on an ongoing basis and includes: generation of output at various levels (from lists to complex processing), ongoing update of data (student and teacher particulars, changes in assignment to study groups), input of data (grades, attendance, and discipline).

\section{THE SURVEY}

A survey was conducted in 1993 and 1994 . The 1994 survey was done in two independent formats: data were gathered from consultants reports and questionnaires were sent out to schools. In both cases the results are similar (but not yet fully analyzed).

It is important to notify that the sample is taken from schools based in townships which signed a contract with the SMIS authority. Those schools receive guidance and are supported by the SMIS authority. The schools are from townships in all districts and represent the Jewish and Arab sectors.

Population: 349 schools :

\section{Implementation stage}

Internalization of administrative module

Use of report generator

Use of pedagogic part

Use analysis of statistical reports for pedagogic purposes

Generate computerized report cards

\begin{tabular}{r}
$\%$ of Sample \\
\hline $98 \%$ \\
$77 \%$ \\
$61 \%$ \\
$42 \%$ \\
$40 \%$
\end{tabular}

\section{CONTRACT WITH THE LOCAL EDUCATION AUTHORITIES}

The Ministry of Education assists local educational authorities who are adopting a township-wide course of action for introduction of SMIS systems into their schools. This assistance covers about $1 / 3$ of the cost of a SMIS Kit which consists of:

- Hardware (computer and printer)

- Software (including maintenance and update)

- SMIS courses for functionaries

- Guidance

- Setup and ongoing operation

Following is the order of activities undertaken upon agreement between the local authority and the Ministry for township-wide deployment:

- Purchase of hardware and software

- Courses for administrative functionaries 
- Guidance backing the setup and ongoing operation

- Institution-wide courses for the teaching staff

By the beginning of the 1993/94 school year, more than 1,400 schools in some 120 local educational authorities throughout Israel had joined the project. Some 60 schools defined as "leading" schools were given increased assistance and were earmarked as demonstration sites for application of the SMIS and as experimental test grounds for new versions of hardware and software,

\section{CONCLUSION}

The School Management Information System improved the handling of information in schools as well as in the Ministry of Education and other educational authorities.

The SMIS authority acts as a coordinator among all existing educational authorities. As there are many of them it is crucial to have an independent body in order to control and coordinate all units involved.

The overall immediate response gives the school an incentive to implement further computerized processes. The ongoing dialogue among the units concerned has improved the quality of data retrieval and management information systems all around.

\section{REFERENCES}

1.Gev Yaffa, (1993) The "MANBAS" - A School Management Information System, G.U.I.D.E 34th Spring Conference

2. Feinmesser A. and Mendel S. (1989) Integrating school in education information systems: Pilot project conclusions (Hebrew) Jerusalem: The Israeli Ministry of Education and Culture.

3. SMIS Standards manual (1992) Jerusalem: the Israeli Ministry of Education and Culture (Hebrew) .

4. Telem M. (1987) The school computer administrator. Educational Technology.

5. Telem M. (1987) Conceptual and operational considerations for the planning and implementation of a pedagogical MIS on a national scale. Programmed learning and educational technology 24,187-193.

6. Ministry of Education, Culture \& Sport (1992) Information on Information; Jerusalem.(Hebrew)

7. Telem M.; Reuron G.; Epstein N. (1985) Pedagogical Management Information Systems in Israel. Report 1: Requirement Survey.

8. Students Manual, Manbas (1992) (Hebrew)

9. Instruction Kit, Manbas (1992) (Hebrew) 